\title{
Revista

\section{RESENHA DESCRITIVA}

\section{Golsorkhi, D., Rouleau, L., Seidl, D. \& Vaara, E. (2010). Cambridge Handbook of Strategy-as-Practice, v.1. UK:}

Cambridge University Press, 349p.

\section{RESUMO}

Desde o artigo seminal de Whittington (1996) propondo que os estudos em Estratégia Organizacional levassem em consideração a estratégia do ponto de vista sociológico, em detrimento somente ao ponto de vista econômico, a abordagem da Estratégia como Prática $(S-a s-P)$, no exterior, parece estar se consolidado. A prova da robustez destes estudos encontra-se no primeiro volume do Handbook de Estratégia como Prática lançado em 2010. O livro, mais do que detalhar estudos passados forneceu (além das direções ontológicas, teóricas e metodológicas) perspectivas alternativas para a expansão e o desenvolvimento futuro da área. Todavia, os estudos da $S$-as- $P$ na América Latina e, em especial, no Brasil parecem, ainda, encontrar dificuldades de compreensão das propostas da abordagem. Desta forma, com o objetivo de contribuir para uma melhor compreensão dos pressupostos ontológicos, teóricos e metodológicos para o aumento da qualidade das futuras pesquisas Brasileiras e Latino Americanas na $S$-as- $P$, devido ao alcance internacional da RIAE, optou-se pela realização de uma resenha descritiva, também conhecida como resenha resumo, do conteúdo do Handbook. Para melhor direcionar este objetivo não foi realizada a resenha da última parte (Parte IV) do livro. Esta seção mostra exemplos de estudos empíricos realizados na área da $S$-as- $P$. Também, existe a intenção futura de se realizar a resenha descritiva do segundo volume do Handbook de Estratégia como Prática publicado em 2015.

Palavras-chave: Estratégia como Prática; Pressupostos Ontológicos; Pressupostos Teóricos; Pressupostos Metodológicos.

\section{ABSTRACT}

Since the seminal paper by Whittington (1996) proposed that the studies in Organizational Strategy consider the strategy by the sociological view, rather than only the economic view, the approach of the Strategy as Practice (S-as-P) abroad it seems to be consolidated. The proof of robustness of these studies was the first volume of Strategy as Practice Handbook published in 2010. The book, more than detailed past studies, provided (in addition the ontological, theoretical and methodological directions) alternative perspectives for expansion and future development of the area. However, studies of S-as-P in Latin America and, in particular, in Brazil seem still find difficulties in understanding the proposals of the approach. Thus, with objective of contributing to a better understanding of ontological, theoretical and methodological assumptions to increase the quality of future research in Brazil and Latin American in the S-as-P approach, due the international range of IJSM, was decided to conduct a descriptive review of the Handbook content. To better orientate the objective the last part of the book (Part IV) was not performed. This section shows examples of empirical studies in S-as-P area. Also there is the future intention to perform a descriptive review of the second volume Strategy as Practice Handbook published in 2015.

Keywords: Strategy as Practice; Ontological Assumptions; Theoretical Assumptions; Methodological Assumptions. 


\section{RESUMEN}

Dado que el artículo seminal de Whittington (1996), que propone que los estudios en Estrategia Organizacional tomaron en cuenta la estrategia del punto de vista sociológico, en lugar de sólo el punto de vista económico, el enfoque de la Estrategia como Práctica (S-as-P) extranjero, parece ser consolidado. La prueba de la solidez de estos estudios es el primer volumen de Manual Práctico de Estrategia y publicado en 2010. El libro más detalles de los estudios anteriores previstas (más allá de las direcciones ontológicas, teóricos y metodológicos) perspectivas alternativas para la expansión y el desarrollo futuro de la zona. Sin embargo, los estudios de S-as-P en América Latina y, en particular, en Brasil también parecen encontrar dificultades para comprender el enfoque propuesto. Por lo tanto, con el fin de contribuir a una mejor comprensión de los supuestos ontológicos, teóricos y metodológicos para aumentar la calidad de la investigación futura de Brasil y de América Latina en el S-as-P, debido a la dimensión internacional de riae, se optó por la realización una revisión descriptiva, también conocido como resumen de revisión, el contenido del Manual. Para orientar mejor este objetivo revisar la última parte no se realizó (Parte IV) del libro. En esta sección se muestran ejemplos de estudios empíricos en el ámbito de la S-as-P. Además, hay una intención de seguir cumpliendo revisión descriptiva de la segunda Estrategia Manual del volumen y de la practica publicada en 2015.

Palabras clave: Estrategia y Práctica; Supuestos Ontológicos; Supuestos Teóricos; Supuestos Metodológicos.

Marcio Luiz Marietto ${ }^{1}$

\footnotetext{
${ }^{1}$ Doutor em Adminsitração pela Universidade Nove de Julho - UNINOVE. Professor e Pesquisador no Programa de Mestrado Profissional em Gestão de Alimentos e Bebidas da Universidade Anhembi-Morumbi/Laureate International Universities. Brasil. E-mail: mlmarietto@uol.com.br
} 


\section{INTRODUÇÃO}

O livro possui quatro organizadores - Damon Golsorkhi, Linda Rouleau, David Seidl e Eero Vaara e contou com mais 36 autores ao longo de seus 21 capítulos. Ele está dividido em quatro partes: I) Questões Ontológicas e Epistemológicas com 6 capítulos; II) Direções teóricas com 6 capítulos; III) Caminhos Metodológicos com 5 capítulos; e IV) Variações de Aplicações com 4 capítulos (esta parte foi excluída desta resenha).

A Introdução do livro, elaborada pelos organizadores, preocupou-se em explicar o que é a Estratégia como Prática enfatizando a análise no micro nível das organizações em detrimento a focar somente os efeitos da estratégia no desempenho econômico das organizações. Esta análise demanda teorias e metodologias diferentes das práticas comuns das pesquisas em estratégia. A $S$-as- $P$ elegeu como conceito chave a "Prática" por entender que as questões centrais como agência, estrutura, ação individual e instituições estão ligadas aos sistemas sociais, cultura e as organizações. Observa-se que é importante notar que as representatividades de diversas escolas do pensamento contribuem para o entendimento do papel central da "Prática" na realidade social. Os autores citaram Filósofos como Wittgenstein (1951), Foucault (1977), Dreyfus (1991), Tuomela (2005), Sociólogos (Giddens 1984; De Certeau 1984; Bourdieu, 1990), Antropólogos (Ortner, 2006), autores da Teoria da Atividade como Vygotsky (1978), Leontiev (1978) e Engeström, et al. (1999), Análise do Discurso (Fairclough, 2003), pesquisadores da Teoria Feminista como (Martin, 2003), além de muitos outros que compõem o corpo teórico e analítico da abordagem. Ao final da introdução, os autores disponibilizaram um apêndice (p.18-20) com os temas e os autores das principais publicações na Estratégia como Prática até aquele momento.

\section{PRIMEIRA PARTE - QUESTÕES ONTOLÓGICAS E EPISTEMOLÓGICAS}

A respeito da proposta ontológica e epistemológica da $S$-as- $P$, a primeira parte do livro propõe a quebra da noção tradicional de que a estratégia é uma propriedade das organizações. A estratégia deve ser entendida como uma atividade ou prática nas organizações, ou seja, a estratégia não é algo que as organizações têm, mas algo que as pessoas nas organizações fazem. Naturalmente, esta transição analítica "do ter" (organização) para "o fazer" (indivíduo) demanda novas propostas ontológicas e epistemológicas para a abordagem.

No primeiro capítulo, Wanda J. Orlikowski distingue três diferentes tipos de práticas: 1) A prática como Fenômeno que estabelece o que acontece na prática na potência de seu acontecimento, ou seja, a prática na conduta diária organizacional estabelecida como um fenômeno interno à organização; 2) A prática como Perspectiva, onde os pesquisadores dirigem suas teorias a uma centralidade na prática (practice-centred) para analisar as estratégias nas organizações; e (3) A prática como Filosofia (Ontologia) concebendo a prática como constituinte da realidade social, ou seja, a vida social constitui-se nas práticas e através das práticas, como tal, sendo vista como uma realização dinâmica da prática em curso.

Neste ponto o livro apresenta uma característica interessante. O segundo e o terceiro capítulo (escritos analogamente por Robert Chia e Andreas Rasche; e Haridimos Tsoukas) tratam de alternativas epistemológicas para a Estratégia como Prática de forma interconectada. Ambos os capítulos buscam estender a terceira proposta do primeiro capítulo alegando ser necessário o debate filosófico (ontologia e epistemologia) para que se possa ir além das abordagens processuais da estratégia.

O segundo capítulo resgata os problemas e as tensões epistemológicas nas pesquisas sobre as práticas estratégicas considerando o legado epistemológico do mundo ocidental deixado pela filosofia grega. Os autores resgatam os conceitos filosóficos de episteme, techné e phronesis para explicar a evolução dos duelos epistemológicos nas pesquisas sociais. Em seguida, os autores, baseados em de Certeau (1984), propuseram dois modos alternativos de engajamento do conhecimento (teorização) que eles nomearam como 'Building' e 'Dwelling'. Por Building os autores entenderam que os pesquisadores supõem existir uma separação inicial pré-cognitiva entre o ator e o mundo, tanto assim, que o ator tem primeiro a necessidade de construir representações mentais e modelos do mundo antes de qualquer envolvimento prático com a realidade. Logo, por Dwelling entenderam que os atores assumem estar intimamente imersos e indissociavelmente interligados com os seus arredores em todas as suas inter-relações complexas. Com isto os autores propõem formas de interligarem estes dois modos epistemológicos e algumas consequências esperadas nas pesquisas da Estratégia como Prática.

No terceiro capítulo o autor sugeriu um debate baseado na onto-epistemologia "Heideggeriana" para a Estratégia como Prática. $\mathrm{O}$ autor explicou que a agência humana é necessariamente exercida no contexto das práticas sóciomateriais. Assim, as atividades particulares dos atores sociais e as práticas sóciomateriais são mutuamente constituídas. Para Heidegger a essência do homem depende de sua relação com o ser e não necessariamente de algum tipo de racionalidade. Portanto, para se instituir uma prática sóciomaterial é necessário penetrar em uma ação cuja identidade é constituída através do uso normativo da linguagem, do corpo e das ferramentas (coisas materiais) disponíveis. Desta forma o autor, baseado na 
filosofia de Heidegger, propôs uma estrutura de pesquisa para a prática estratégica com três diferentes tipos de ações de acordo com a forma de envolvimento e o grau de intencionalidade: 1) a habilidade prática (baseada no entendimento tácito) que constitui a ação não deliberada; 2) a habilidade deliberada (baseada na consciência explicita; e 3) o enfrentamento deliberado (baseado em uma consciência temática) que é a forma mais deliberada de ação. A contribuição desta estrutura para a $S$-as- $P$ reside em compreender como o pensamento estratégico ou mesmo o strategizing podem ser observados como habilidades de lidar com as dificuldades práticas por meio da utilização da linguagem, do corpo ou mesmo de ferramentas disponíveis no contexto situacional.

O quarto capítulo trata da epistemologia construtivista nos estudos da $S$-as- $P$. Simon Grand, Johannes Rüegg-Stürm e Widar von Arx após explicarem os três programas construtivistas (construtivismo social, construtivismo sistêmico e o empirismo construtivista) abordaram os impactos que o construtivismo pode gerar nos estudos da $S$-as- $P$. Eles sugerem estudos com bases epistemológicas construtivistas na construção das práticas de strategizing, na constituição da prática estratégica, ou seja, na criação da estratégia e na construção da pesquisa estratégia evidenciando a construção integrada de uma estrutura que relate as teorias predominantes, as metodologias e os temas existentes entre o construtivismo e as pesquisas em estratégia.

Para evidenciar como os estudos baseados na Estratégia como Prática contribuíram para o campo dos estudos organizacionais, Karen Golden-Biddle e Jason Azumado mapearam as contribuições empíricas no quinto capítulo do Handbook. Baseados nos estudos de Locke e Golden-Biddle (1997), os autores selecionaram 26 trabalhos empíricos, na área da $S$-as$P$, publicados no Administrative Science Quarterly e no Academy of Management Journal. As análises transcorreram por duas dimensões: 1) Uma dimensão que busca sintetizar a coerência das pesquisas relatando as conexões entre os estudos; e 2) uma dimensão que relata as incoerências entre as pesquisas revelando as contradições e as discordâncias entre os autores dos estudos. Os autores constroem uma matriz (p.86) que revela, nas células vazias, oportunidades importantes para futuras contribuições na área da Estratégia como Prática.

No capítulo final da primeira parte do livro Ann Langley buscou explicar como os pesquisadores no campo da $S$-as- $P$ podem construir um corpo acumulativo de conhecimento, uma vez que, a abordagem favorece pesquisas com amostras menores e análises microscópicas. Um dos objetivos da abordagem é conhecer os procedimentos das pessoas que formulam e trabalham nas estratégias para as empresas. Isto é diferente das técnicas já estabelecidas nos estudos anteriores da estratégia na visão econômica. Parece ser ilusório esperar que a pesquisa em estratégia de nível micro, explique os resultados do desempenho organizacional. A questão relevante parece ser sobre o que sabemos sobre as práticas dos que estabelecem as estratégias e o que poderemos fazer para evidenciá-las? A autora explica que os resultados devem ser relacionados às práticas dos estrategistas para a eficácia das ações propostas. Portanto, o ponto de partida a ser analisado são os fenômenos e não uma teoria em particular. O pesquisador deve descobrir o conhecimento dos praticantes, torná-lo explícito e disponível para os outros. Para isto pode-se utilizar uma grande variedade de teorias de base social e voltadas para "prática" (theories of practice - p.96) para explicar as possíveis variâncias dos fenômenos e, assim, acumular e expandir o conhecimento da abordagem.

\section{SEGUNDA PARTE - DIREÇÕES TEÓRICAS}

A segunda parte do livro retratou as perspectivas teóricas que se adaptam aos pressupostos ontológicos e epistemológicos discutidos na primeira parte. Os capítulos enquadram as teorias de diferentes escolas como a sociologia, psicossociologia, entre outras, em função das pesquisas na área de gestão. Os autores preocuparam-se, além de explicar os pressupostos teóricos, em demonstrar como estas lentes teóricas podem ser aplicadas nas práticas da gestão estratégica. A intenção é de elucidar as abordagens especificas que fornecem sentido aos processos, as atividades, as práxis, ao strategizing e aos practitioners durante os processos e atividades diárias de tomada de decisão estratégica.

No sétimo capítulo do Handbook e primeiro capítulo da segunda parte Richard Whittington ofereceu diversas sugestões para os estudos da $S$-as- $P$ na perspectiva Estruturacionista (Giddens, 1984). Para a Estratégia como Prática a Teoria Estruturacionista oferece aos pesquisadores vários elementos atraentes como: a) atenção aos detalhes micro sociológicos; b) sensibilidade ao contexto institucional; e c) abertura à mudança. A literatura emergente da Estratégia como Prática já adotou aspectos da Teoria da Estruturação. No capítulo foi realizada a revisão, de forma sintetizada, de oito estudos empíricos extraídos de Journals Americanos e Europeus que utilizaram, de forma substancial, as noções Giddens dentro da tradição da $S$-as- $P$ revelando, assim, alguns pressupostos Estruturacionistas já conectados com a $S$ $a s-P$. O autor explicou que os pesquisadores da $S$ - $a s-P$ claramente encontrarão utilidade para a Análise da Conduta Estratégica no fenômeno da Dualidade da Estrutura Organizacional, especialmente para a compreensão do Poder de Agência dos gestores. Dada à audiência dos pesquisadores da $S$-as- $P$ este fluxo de trabalho tem forte potencial de desenvolver conhecimento para fora do topo hierárquico podendo envolver consultores e outros atores sociais que lidam com a estratégia nas Organizações. $\mathrm{O}$ autor concluiu o capítulo com sugestões para estudos futuros na área. 
No segundo capítulo da segunda parte e oitavo capítulo do livro Paula Jarzabkowski foca na Teoria da Atividade (Vygotsky, 1978; Leontiev, 1978) como base para a pesquisa na $S$-as- $P$. A autora tenta relacionar o conceito de mediação entre o sujeito coletivo (gerentes de alto escalão), a comunidade e as atividades direcionadas ao resultado por meio das práticas de mediação situadas na construção da atividade, transformando esta mediação em nível de análise. Em outras palavras, as ações estratégicas são partes dos sistemas de atividades que compreendem os atores e a comunidade social em que eles interagem. A mediação, entre estes atores sociais e a comunidade ao qual estão inseridos, é realizada por meio do uso das ferramentas materiais e simbólicas ligando-se, assim, os níveis de análise chaves da $S$-as-P: práxis, prática e practitioners. A autora comparou as teorias da atividade com as teorias da prática e, também, conclui o capítulo sugerindo mais pesquisas em profundidade na gestão estratégica inspiradas na Teoria da Atividade.

A Teoria da Prática de Pierre Bourdieu foi retratada no nono capítulo do livro por Marie-Léandre Gomez. Os estudos de Bourdieu sobre a prática representam uma contribuição importante ao campo das ciências sociais. Bourdieu desenvolveu uma visão sistêmica da prática que aborda as relações entre os agentes com o campo e as disposições para a ação. Em tese, a prática é o fazer dos atores sociais que possui significado e valor em um campo particular, sendo que a noção que caracteriza o campo é a autonomia de certo domínio de concorrência e disputa interna entre os agentes. Este fazer reflete a trajetória social dos agentes através da sua posição no campo. Esta posição no campo está relacionada ao capital possuído (não apenas capital econômico, mas também cultural e social), seus habitus (percepções e ações que traduzem estilos de vida, julgamentos políticos, morais, estéticos, entre outros), disposição para agir, predisposições e crenças que são constituídas durante a experiência passada em um campo particular. Desta forma, os conceitos de campo, capital, habitus e práticas formam um sistema. Este sistema é responsável para a prática por meio das dimensões individuais e sociais em sua intencionalidade, também representa as limitações estruturais, a racionalidade e os elementos não cognitivos. A autora explica que os pressupostos teóricos de Bourdieu servem, também, como alternativa teórica para se superar os dilemas entre micro e macro e entre agência e estrutura promovendo um melhor entendimento entre as diversas características da falsa dicotomia entre estratégia e strategizing. Vale ressaltar que no primeiro capítulo desta parte elaborado por Richard Whittington, o mesmo evidencia que a teoria da Prática de Bourdieu é uma teoria concorrente ao Estruturacionismo de Giddens.

Saku Mantere propôs à Estratégia como Prática, no décimo capítulo do livro e quarta parte das perspectivas teóricas, a abordagem filosófica de Wittgenstein. $\mathrm{O}$ autor explicou que a abordagem
Wittgenstein influenciou a "practice turn" das ciências sociais, além de Giddens e Bourdieu terem sido, também, muito influenciados pelo mesmo. Para Mantere a percepção de Wittgenstein sobre os "Jogos de Linguagem" pode contribuir de forma relevante a $S$ $a s-P$. Estes jogos de linguagem compõem-se de um conjunto de padrões de atividades (discursivas ou não discursivas) que só pode ser exercido se os atores sociais aderirem a um determinado conjunto de regras pré-estabelecidas. Esta linguagem é concebida por meio da representação de mundo de forma significativa pelos atores sociais, sendo que o uso da linguagem está na raiz da prática social de diversas maneiras, além de ser utilizada em diversos tipos de comunidades. Esta estratégia discursiva pode focar em diversos aspectos ainda negligenciados nos estudos de estratégia. O autor conclui que, por exemplo, analises de conversas relativas às estratégias podem prover ilustrações concretas da vida real dos estrategistas nas organizações.

Para Florence Allard-Poesi a perspectiva de Foucault, que foi apresentada no décimo primeiro capítulo do Handbook, pode evidenciar novos caminhos para se observar os fenômenos sociais na $S$ as- $P$. Foucault pode favorecer a dimensão discursiva da gestão estratégica, uma vez que a análise se debruça sobre um conjunto heterogêneo de discursos e práticas materiais. A autora explicou que Foucault analisa, por meio do discurso, os conjuntos de práticas humanas referindo-se ao que os atores sociais fazem e a maneira como o fazem. Na visão de Foucault aquele que pratica algo é constituído por meio das práticas discursivas e não discursivas que ele realiza. Estas práticas revelam as técnicas pelas quais os homens buscam governar o mundo, influenciando, alimentando e desafiando uns aos outros, sendo que a estrutura desses diferentes conjuntos de práticas, suas possíveis junções e disjunções, raramente, são consideradas por pesquisas na gestão estratégica. Estas práticas discursivas e materiais são governadas por regras especificas que estruturam aquilo que pode ser lido, dito e feito em torno da estratégia. Destarte, a autora concluiu propondo que a justaposição da perspectiva de Foucault e a abordagem atual da estratégia como prática revelam elementos contrastantes da estratégia, estrategistas e suas ambições abrindo caminho para novas áreas de investigação.

No último capítulo das direções teóricas do Handbook, uma perspectiva interessante foi sugerida por Valérie-Inès De La Ville e Eléonore Mounoud sobre observar a estratégia por meio dos textos e narrativas. As autoras chamam a atenção para elementos que normalmente não são esclarecidos, não ditos e, muitas vezes inconscientes, que constituem a prática da estratégia e do strategizing. Estes elementos habitam nas pessoas, eventos e nas ferramentas convencionais que se localizam na formulação e na implementação da estratégia. As autoras se apoiam nos trabalhos de Michel de Certeau e Paul Riceour, onde focam na escrita e leitura de textos e narrativas nas 
atividades diárias da estratégia nas organizações. Elas entendem que uma conversa que incorpora, de forma recursiva, outra conversa pode ser entendida como meta-conversação e esta prática discursiva é algo que as organizações fazem diariamente de forma intrínseca e idiossincrática podendo revelar detalhes, muitas vezes, encobertos (por isso a necessidade de análise dos meta-discursos) durante os episódios de strategizing, assim ofereceram um modelo de "Elaboração da estratégia a partir de textos e narrativas" (p.193). As autoras concluem que a incorporação teórica dos pressupostos textuais e discursivos da estratégia pode desafiar e revelar lados ocultos diferentes das visões formais da estratégia, uma vez que tendem a revelar aquilo que é dito dentro do que é feito.

\section{TERCEIRA PARTE - CAMINHOS METODOLÓGICOS}

A terceira parte do Handbook pontua os desafios metodológicos impostos a abordagem da $S$-as$P$. Já percebemos, na primeira parte do livro, que a abordagem compreende uma estrutura ontoepistemológica diferente da abordagem econômica da Estratégia, logo na segunda parte o livro sugeriu teorias que acompanham esta tendência de analise. Naturalmente, a terceira parte sugere direções metodológicas que possam superar o desafio de proximidade do pesquisador que deve atuar, simultaneamente, próximo ao fenômeno da prática e das atividades estratégicas, em geral de forma longitudinal, empregando uma lente teórica, em geral complexa, e ao mesmo tempo fazendo uso das ferramentas metodológicas. Nesta terceira parte os organizadores sugeriram que para se observar as práticas são necessários caminhos "metodologicamente inovadores" e estes caminhos estão dispostos nos capítulos desta terceira parte.

Inaugurando o primeiro capítulo da terceira parte e décimo terceiro capítulo do livro foram propostos métodos que estão na fronteira do conhecimento metodológico para suportarem os novos insights da Estratégia como Prática. Anne Sigismund Huff, Anne-Katrin Neyer e Kathrin Möslein iniciam o capítulo recuperando a crise econômica de 2008 para evidenciar que deve haver uma expansão na agenda metodológica da estratégia, especificamente no strategizing, para preencher as lacunas destes macro eventos. Estas metodologias devem ser embasadas na ontologia e na epistemologia proveniente do envolvimento de agentes (decisores) políticos, profissionais e acadêmicos de outras disciplinas que além de definir as ferramentas de coleta de dados, com ênfase nas abordagens etnográficas, devem, também, adotar novos métodos que mais eficazes em revelar padrões de atividades em grandes organizações. No decorrer do capitulo as autoras apontaram as deficiências do método etnográfico, porém indicaram que ele continua sendo o mais eficaz e sugeriram cinco características desejáveis para as coletas de dados: 1) Aumento da atual capacidade (quantidade) de coleta e análise de dados; 2) Aumento da variedade de dados coletados e analisados; 3) Entendimento contextual das características (e/ou contextos) dos sistemas; 4) Aumento da generalização de resultados; e 5) Aumento da precisão da coleta e análise dos dados. As autoras finalizaram incentivando os pesquisadores a adotarem métodos que ajudem explicar melhor os fenômenos de strategizing, de tal modo que, possam ajudar no desenvolvimento da área da $S$ - $a s-P$.

Eero Vaara ilustrou a Análise do Discurso Crítico como uma metodologia para a $S$-as- $P$ no décimo quarto capítulo do livro. $\mathrm{O}$ apelo recai sobre como examinar o papel característico dos discursos sobre os elementos práticos e diários da estratégia e do strategizing. Em outras palavras, o autor procurou, ao longo do capítulo, expor o papel central dos textos na estratégia formal; a construção discursiva das concepções de estratégia e a subjetividade na elaboração de estratégias organizacionais; os processos de legitimação através do discurso estratégico; e as bases ideológicas dos mesmos como um corpo constitutivo do conhecimento e da práxis estratégica. Ao final, o autor concluiu que a Análise do Discurso Crítico pode acomodar várias perspectivas teóricas e métodos empíricos, sendo que por meio destas análises podemos compreender melhor a ligação entre a prática discursiva e as práticas estratégicas e, também, as consequências sociais do discurso estratégico.

A contribuição da Etnometodologia e da Análise de Conversação para a pesquisa do strategizing na $S$ - $a s-P$ é retratada no terceiro capítulo da parte sobre metodologia do Handbook. Os estudos etnometodológicos possuem destaque nos trabalhos Harold Garfinkel (1967) que foi influenciado pela fenomenologia das obras de Husserl e Schutz na década de 1940. O pressuposto fenomenológico preocupa-se com a atividade incorporada e a produção prática cotidiana de tomada de mundo. Esta atividade esta contida no detalhe da conversa concreta e no comportamento que os atores sociais coproduzem. Dalvir Samra-Fredericks, autora do capítulo, percebeu que se "tomada de mundo" fosse substituído por "elaboração da estratégia" haveria um vasto campo de interconexão empírica para a Estratégia como Prática que examinaria o trabalho dos estrategistas, isto é, a atividade incorporada e coproduzida, a fala concreta e o comportamento social no qual constituem a ordem social e os sentidos do quotidiano. Em outro ponto, a autora recupera Garfinkel e Sacks (1970) para evidenciar que os autores inauguraram a Análise de Conversação que possui como premissa a gravação de conversas e interações gerando transcritos que são analisados em um sistema de notação pormenorizada. $\mathrm{Na}$ análise de conversação o contexto não é assumido ou tratado como "dado", mas, sim, uma criação dinâmica que é expressa na (e através da) organização sequencial de interação das práticas dos atores sociais 
para a realização de uma ordem social. Para expor seus pontos a autora revisa um estudo organizacional, de sua autoria, que utilizou ambas as metodologias. O capítulo finalizou com alertas sobre os desafios de se realizarem estudos na $\mathrm{S}$-as-P utilizando-se estas metodologias, todavia, eles tendem a ser teoricamente significativos, uma vez que, revelam detalhes que exibem propriedades e detalhes de ordem social em sequenciamento continuo e cotidiano.

A proposta de que os practitioners da estratégia e suas práticas fossem examinadas através das lentes dos estudos de Identidade figura no quarto capítulo da terceira parte e decimo sexto capítulo do Handbook. O apelo de Phyl Johnson, Julia Balogun e Nic Beech advém da necessidade de se pesquisar os estrategistas com um olhar muito mais próximo do pesquisador em relação ao objeto de sua pesquisa. Este olhar deve permitir penetrar o suficiente para detalhar a atividade estratégica e, principalmente, quem são estas pessoas e quais são seus hábitos durante a execução destas atividades. Os autores utilizaram um exemplo empírico para ilustrar a proposta de análise da identidade dos estrategistas no impacto de seus trabalhos diários. Após, explicaram os conceitos de identidade que acreditam estarem mais próximos das pesquisas para a $S$-as- $P$ que, em geral, podem ser extraídos por meio das análises de narrativas dos practitioners em estudos longitudinais. Ao final propuseram uma agenda colaborativa que agregue os estudos de identidade aos da $S$ - $a s-P$.

O último capítulo da parte metodológica e décimo sétimo capítulo do livro recomendou o estudo do strategizing pela coleta e análise de dados por meio de um tipo especifico de estudo biográfico - As Narrativas das Práticas. A narrativa das práticas é um método especifico das histórias de vida que foca na experiência de trabalho e na trajetória do profissional. Este método permite ao pesquisador "escavar" o modo de vida dos atores sociais como CEOs, TMTs, gerentes, entre outros para capturar aquilo que é dado como certo (taken-for-granted) em suas rotinas, eventos e interações que constituem suas práticas profissionais. Ao longo do capítulo, Linda Rouleau ilustrou suas experiências com este método quando examinou como os gerentes de uma organização lidaram com os processos de reestruturação. Ao final do capitulo a autora discute os desafios e os benefícios de se utilizar este método nos estudos da $S$-as- $P$.

\section{COMENTÁRIOS FINAIS}

Esta resenha teve o objetivo de resumir os capítulos das três primeiras partes (pressupostos ontológicos, teóricos e metodológicos) do primeiro volume do Handbook de Estratégia como Prática para possibilitar um aumento na compreensão das propostas da área pelos pesquisadores Latino Americanos e Brasileiros. Nossa intenção, no futuro, é de realizar a resenha descritiva, também, do segundo volume do
Handbook de Estratégia como Prática. Com o objetivo de ampliar a compreensão da área e aumentar a qualidade das pesquisas na $S$-as- $P$ no Brasil e na América Latina, a resenha do segundo volume do Handbook deverá vir acompanhada com alguns comentários generalistas sobre algumas inconsistências e incoerências encontradas nos estudos sobre $S$ - $a s-P$ publicados no Brasil. Naturalmente, estes comentários ocorrerão à luz dos livros resenhados.

\section{REFERÊNCIAS}

Bourdieu, P. (1990) The Logic of Practice. Cambridge: Polity Press.

De Certeau, M. (1984) The Practice of Everyday Life. Berkeley: University of California Press.

Dreyfus, H. (1991) Being-in-the-World. Cambridge, MA: The MIT Press.

Engeström, Y., Miettinen, R. \& Punamäki, R-L. (eds.) (1999) Perspectives on Activity Theory. Cambridge: Cambridge University Press.

Fairclough, N. (2003) Analyzing Discourse: Textual Analysis for Social Research. London: Routledge.

Foucault, M. (1977) Discipline and Punish. The Birth of the Prison. New York: Pantheon Books.

Garfinkel, H. (1967) Studies in ethnomethodology. 2ed. Englewood Cliffs, NJ: Prentice Hall.

Garfinkel, H. \& Sacks, H. (1970) 'On formal structures of practical actions'. In J. D. Mckinney \& E. A. Tiryakian (eds) Theoretical sociology. New York: Appleton-Century Crofts, 337-366.

Giddens, A. (1984) The Constitution of Society. Oxford: Polity Press.

Leontiev, A. N. (1978) Activity, Consciousness and Personality. Englewood Cliffs: Prentice-Hall.

Locke, K. \& Golden-Biddle, K. (1997) Constructing Opportunities for Contribution: Structuring Intertextual Coherence and "Problematizing" in Organizational Studies. Academy of Management Journal, 40(5): 1023-1062.

Martin, J. (2003) Feminist theory and critical theory: Unexplored synergies. Studying Management Critically, 66-91.

Ortner, S. B. (2006) Anthropology and Social Theory. Culture, Power and the Acting Subject. Durham: Duke University Press. 
Tuomela, R. (2005) The Philosophy of Social Practices. A Collective Acceptance View. Cambridge: Cambridge University Press.

Vygotsky, L. (1978) Mind in Society: The Development of Higher Psychological Processes. Cambridge, MA: Harvard University Press.

Whittington, R. (1996) Strategy as practice. Long Range Planning: 29(5): 731-735.

Wittgenstein, L. (1951) Philosophical Investigations. Oxford: Basil Blackwell. 